\title{
Zs Research fuare \\ Determination of reference genes as a quantitative standard for gene expression analysis in mouse mesangial cells stimulated with TGF- $\beta$
}

Bruno Aristides dos Santos Bronel

Universidade Federal de São Paulo

Ana Carolina Anauate

Universidade Federal de São Paulo

Edgar Maquigussa

Universidade Federal de São Paulo

Mirian Aparecida Boim

Universidade Federal de São Paulo

Antônio Silva Novaes ( $\square$ antonio.novaes52@gmail.com )

Universidade Federal de São Paulo

\section{Research Article}

Keywords: Housekeeping genes, Reference genes, Mesangial cells, Transforming growth factor- $\beta$, Reverse transcriptionquantitative polymerase chain reaction

Posted Date: February 7th, 2022

DOI: https://doi.org/10.21203/rs.3.rs-1325483/v1

License: (c) (i) This work is licensed under a Creative Commons Attribution 4.0 International License. Read Full License 


\section{Abstract}

Reverse transcription-quantitative polymerase chain reaction (RT-PCR) is the gold standard technique for gene expression analysis, but the choice of quantitative reference genes (housekeeping genes, HKG) remains challenging. Identification of the best HKG is essential for estimating the expression level of target genes. The aim of this study was to identify the best HKG for an in vitro model with mouse mesangial cells (MMCs) stimulated with $5 \mathrm{ng} / \mathrm{mL}$ of TGF- $\beta$. Candidate five HKG were selected: Actb, Hprt, Gapdh, 18 S and Ppia. After quantitative expression, the best combination of these genes was analyzed in silico using six software programs. To validate the results, the best genes were used to normalize the gene expression of fibronectin, vimentin and a-SMA. In silico analysis revealed that Ppia, Gapdh and $18 S$ were the most stable genes between the groups. GenEX software and Spearman's correlation determined that combination of Ppia and Gapdh is the best HKG pair. Validation of the HKG by normalizing fibronectin, vimentin and a$S M A$ were consistent with results from the literature. This study established the combination of Ppia and Gapdh as the best HKG pair for gene expression analysis by RT-PCR in this in vitro model using MMCs treated with TGF- $\beta$.

\section{Background}

Transforming growth factor- $\beta$ (TGF- $\beta$ ) is a major renal profibrogenic cytokine and plays a critical role in mesangial dysfunction in many pathophysiological conditions characterized by excessive accumulation of extracellular matrix (ECM) proteins, mesangial cell (MCs) hypertrophy, and proliferation ${ }^{1,2}$. The interaction of TGF- $\beta$ and its receptors forms a heterodimeric complex, which is translocated into the nucleus and regulates transcription of target genes, such as the genes coding for fibronectin, vimentin and a-smooth muscle actin (a-SMA) ${ }^{1,3-5}$.

The reverse transcription-quantitative polymerase chain reaction (RT-PCR) is the gold standard method to identify changes in mRNA expression levels ${ }^{6-8}$. Considering the many steps of RT-PCR, and that several factors can influence expression levels, the normalization of target genes is crucial for accurate gene expression quantification. Currently, the most accepted method of target gene expression normalization by RT-PCR technique is through quantification of very stable endogenous housekeeping genes (HKG) ${ }^{9,10}$.

HKG or the reference genes, are genes required for maintenance of basal cellular functions ${ }^{11,12}$ and the ideal reference genes are expected to be expressed in all cell types and should show minimal variation in the expression, regardless of cell cycle state, developmental stage, external stimuli and physiological condition ${ }^{10,12,13}$. Examples of the most used reference genes include Actin beta and Gapdh ${ }^{14}$. Although the use of HKG is the most accurate method for normalizing mRNA expression levels, it is well known that the expression levels of even the most stable HKG can change depending on cell type and experimental conditions and design ${ }^{8,15}$.

Despite the growing number of studies investigating the reference genes for renal disease models ${ }^{10,16,17}$, at present there are no detailed reports that select the most stable gene recommended for the frequently used in vitro model of TGF- $\beta$-induced fibrogenesis in MCs. This limitation may lead to non-reproducible data. The present study aimed to evaluate the performance of five frequently used reference genes (Actb, Hprt, Gapdh, $18 S$ and Ppia) in renal models ${ }^{18-}$

${ }^{29}$ and to identify the most stable ones and the optimal number of genes for normalization the expressions of target genes by RT-PCR in MCs treated with TGF- $\beta$ model.

\section{Results}

\section{Candidate housekeeping genes}


First, we followed a stepwise strategy to identify the best HKG expression by RT-PCR analysis. The workflow diagram is shown in Fig. 1. The samples were classified into three groups: (1) control cells $(n=6)$; $(2)$ cells treated with TGF- $\beta$ ( $n=$ 6); and (3) All, which includes all cells $(n=12)$. The Ct values of the five candidate HKG ranged between 23.511-9.387 and are expressed as [median (interquartile range)]. Ct values are inversely proportional to gene expression, and the mean highest Ct value among the candidate genes was achieved by Hprt [20.876 (2.05)], indicating the least expressed gene. In contrast, the lowest Ct value was obtained for $18 S$ [10.232 (0.50)], indicating the highest expression among the candidate genes. Gapdh [17.948 (2.41)], followed by Actb [15.986 (2.44)] and Ppia [15.514 (1.54)], showed moderate expression. The median Ct values of triplicate reactions according to each gene is shown in Fig. 2. Furthermore, no statistically significant differences were found between the control group and the TGF- $\beta$-treated group, these data suggest that the endogenous genes selected showed good stability and that their expression did not change when treated with TGF- $\beta$.

\section{Stability analysis of housekeeping genes}

Stability values of the five candidate HKG were determined using six software packages (Supplementary Table 1). Genes with the smallest stability value (SV) had the most stable expression. Following NormFinder criteria $(S D<0.5)$, only Ppia and Gapdh had a SD value below 0.5 in the All and control groups, while in the TGF- $\beta$-treated group, only Ppia respects this criterion (Table 1 and Supplementary Table 1). The GeNorm software defines an $\mathrm{M}$ value $<1.5$; therefore, the genes with lower variability were the following: Hprt, 18S and Ppia for All samples; Gapdh, Ppia, Hprt and 18S for the control group; and 18S, Hprt and Ppia for the TGF- $\beta$-treated group (Table 1 and Supplementary Table 1). According to Bestkeeper criteria, when considering all three experimental groups, only $18 S$ fits the parameters, although the CV exceeds 3.0 in the TGF- $\beta$ group (Table 1 and Supplementary Table 1).

In the RefFinder and $\Delta$ Ct method analysis, the most stable gene in the All group was Ppia, while in the control group it was Gapdh. However, in the TGF- $\beta$ group, the most stable genes were $18 S$ and Ppia according to RefFinder and $\Delta$ Ct method analysis, respectively (Table 1 and Supplementary Table 1). The evaluation of the most stable gene on DataAssist software identified that Ppia as the best HKG in the All, control, and TGF- $\beta$-treated groups (Table 1 and Supplementary Table 1). In all software analysis, the less stable gene was Actb (Supplementary Table 1). Based on software analysis and a qualitative inspection of all ranks generated, the best HKG for the All group was Ppia, whereas it was Gapdh for the control group and Ppia or $18 S$ for the TGF- $\beta$-treated group (Table 1).

Table 1

Best housekeeping gene for each group of samples yielded by software analysis.

\begin{tabular}{|llllllll|}
\hline Groups & NormFinder & GeNorm & RefFinder & DCt method & Bestkeeper & DataAssist & Best HKG \\
\hline All & Ppia & Hprt/18S & Ppia & Ppia & $18 S$ & Ppia & Ppia \\
\hline Control & Gapdh & Gapdh/Ppia & Gapdh & Gapdh & $18 S$ & Ppia & Gapdh \\
\hline TGF- $\boldsymbol{\beta}$ & Ppia & 18S/Hprt & $18 S$ & Ppia & $18 S$ & Ppia & Ppia or 18S \\
\hline All, all samples. HKG, housekeeping genes. & & & & \\
\hline
\end{tabular}

\section{Analysis of the best combination of housekeeping genes}

The GeNorm software recommends two genes as the best HKG. Table 2 shows the best combination of HKG for each group/software package. In the All group, the best pair of HKG is Ppia+Gapdh or Ppia+18S, in the control group is Gapdh+Ppia and in the TGF- $\beta$ group is Ppia+18S (Table 2). To analyze the effects of the best candidate HKG, the expression levels of the top three (Ppia, Gapdh, and 18S) were normalized by each other (Fig. 3). All comparisons 
showed no statistically differential expression, meaning that Ppia, Gapdh, and $18 S$ did not differ between the groups when normalized by each other (Fig. 3).

Table 2

The best combination of housekeeping genes for each group of samples yielded by software analysis.

\begin{tabular}{|c|c|c|c|c|c|c|c|}
\hline Groups & NormFinder & GeNorm & RefFinder & $\Delta \mathrm{Ct}$ method & Bestkeeper & DataAssist & $\begin{array}{l}\text { Best HKG } \\
\text { Pair }\end{array}$ \\
\hline All & Ppia/Gapdh & Hprt/18S & Ppia/18S & Ppia/Gapdh & 18S/Hprt & Ppia/Gapdh & $\begin{array}{l}\text { Ppia/Gapdh } \\
\text { or Ppia/18S }\end{array}$ \\
\hline Control & Gapdh/Ppia & Gapdh/Ppia & Gapdh/Ppia & Gapdh/Ppia & 18S/Hprt & Ppia/Gapdh & Gapdh/Ppia \\
\hline TGF- $\beta$ & Ppia/Gapdh & 18S/Hprt & 18S/Ppia & Ppia/18S & 18S/Hprt & Ppia/Gapdh & Ppia/18S \\
\hline
\end{tabular}

\section{Determination of the suitable number of housekeeping genes}

After rating the candidate HKG by their stability values, the optimal number of candidate genes to be used in each dataset must be established. This analysis was performed in the GenEx software package and using the Acc.SD for calculating the optimal number of reference genes. The results showed that one gene (Ppia or 18S) is the optimal number of HKG for normalization of gene expression in TGF- $\beta$-treated samples (Fig. 4). Two genes are required for normalization in the All group (Ppia+Gapdh or Ppia+18S) and control group (Gapdh+Ppia) (Fig. 4).

\section{Correlation between the top three candidate housekeeping gene expressions}

Correlation analysis were performed using the gene expression data from all samples. The expression levels of the three best candidate HKG showed a strong correlation between Ppia and Gapdh ( $\rho=0.804, \mathrm{p}=0.002)$ (Fig. 5). Also, no statistically significant correlation was found between Ppia and $18 S(\rho=-0.392, \mathrm{p}=0.208)$ and Gapdh and $18 S(\rho=$ $-0.580, p=0.052$ ) (Fig. 5). These results suggest that other than the fact that Ppia and Gapdh showed a strong correlation, they are correlated in all the samples and can be used together as suitable HKG.

Validation of the best candidate housekeeping genes for normalizing target genes of fibronectin, vimentin, and a-SMA

To validate the stability of the top three candidate HKG, the relative expression of fibronectin, vimentin, and $a$-SMA target genes was normalized using different combinations of Ppia, Gapdh and 18S(Fig. 6). The expression levels of fibronectin, vimentin, and $a-S M A$ target genes were consistent with upregulation in the TGF- $\beta$ group relative to controls (Fig. 6). The normalization of target genes by the two less stable genes (Actb and Hprt) was also evaluated (Supplementary Fig. 1). The use of these HKG, whether alone or in combination, was not able to demonstrate the statistically significant difference that was expected between controls and TGF- $\beta$-treated samples of fibronectin and $a$ SMA (Supplementary Fig. 1).

\section{Discussion}

The broadly accepted method used to normalize gene expression through RT-PCR technology involves the expression of endogenous HKG. However, the utility of HKG must be validated for specific experimental conditions, since the expression of these endogenous genes can vary depending on experimental conditions ${ }^{8-10,15}$. In vitro systems, including cultured MCs, constitute an useful model to study many pathophysiological states affecting the glomeruli, such as glomerulosclerosis ${ }^{1}$. Therefore, we aimed to determine the most stable reference genes for mRNA 
quantification in studies performed in vitro, mimicking the in vivo glomerular fibrosis using MMCs treated with TGF- $\beta$ $10,13,15,30$.

Since each algorithm ranked the best candidate HKG, the software packages recommended Ppia, Gapdh and 18 Sas the most stable reference genes between the groups. Peptidylprolyl isomerase A (Ppia), a highly abundant protein in the cytoplasm, takes part in various intracellular functions, including a homeostatic role in protein folding, protein trafficking, intracellular signaling, transcription, inflammation, apoptosis, and regulation of activity of other proteins ${ }^{31-}$ ${ }^{33}$. Glyceraldehyde-3-phosphate dehydrogenase (Gapdh) catalyzes the sixth reaction of anaerobic glycolysis, which produces ATP and pyruvate. Other than metabolic functions, this enzyme has been implicated in non-metabolic processes, such as apoptosis induction, DNA repair, cellular proliferation, and transcriptional activation ${ }^{34-36}$. Small subunit 18S ribosomal RNA (18S) is the small component of eukaryotic cytoplasmic ribosomes and is one of the molecular markers ${ }^{37,38}$.

The other two genes considered in this study (Hprt and Actb) are also known as common reference genes. Hypoxanthine phosphoribosyltransferase $(H p r t)$ is responsible for purine metabolism, and deficiency of this gene dysregulates cell cycle-controlling functions and cell proliferation mechanisms ${ }^{39,40}$. Actin beta (Actb), which is highly abundant in eukaryotic cells, is essential for a variety of cellular functions and is involved in maintaining the cell's structure, integrity, and motility ${ }^{41}$. Although extensively used as reference genes ${ }^{14}$, Hprt and Actb ranked as the least stable in this study; however, further studies are needed to better delineate the interactions of these genes with TGF- $\beta$.

Since Ppia, Gapdh, and $18 S$ were the most suitable candidate reference genes, we normalized them by each other, resulting in no statistically significant differences between groups, which suggests that these genes are good choices for our experimental conditions. After determining the candidate HKG by their stability values, we established the optimal number of reference genes using GenEx software. According to calculated Acc.SD, the optimal number of HKG in this model is the combination of two genes. When used together, Ppia and Gapdh showed a strong correlation, indicating that all samples were correlated and validating the best pair of HKG.

It is well demonstrated that TGF- $\beta$ stimulates production of fibronectin, vimentin, and $a$-SMA in cultured MCs ${ }^{2,42}$; thus, the best HKG combinations obtained in the present analysis were used to normalize these target genes. Several studies have reported that Ppia ${ }^{14,17,43-48}$, Gapd $h^{44,49,50}$, and $18 S^{45,51-53}$ are suitable reference genes and could be used as normalizers of target genes in different models. In the present study, the top three candidate reference genes, whether used alone or in combination, showed the expected increase in the expression of the target genes in the TGF- $\beta$-treated group. In contrast, the less stable HKG, employed alone or in combination, did not yield these expected differences, indicating that the in silico analysis selected the better, more stable HKG for this in vitro fibrosis model; they also revealed that an inadequate choice of the endogenous standard HKG could influence the results.

It is important to mention that other non-tested genes in the preset study can also be used for normalization of the expression of target genes, and additional studies are needed to identify additional candidate genes. Furthermore, this study is specific to MMCs stimulated with TGF- $\beta$ and, thus, the conclusions drawn from our study are not transferable to other models that employ MMCs.

Among the reference genes tested in this study, the combination of Ppia and Gapdh was the best HKG pair and should, therefore, be used as HKG in gene expression analysis in TGF- $\beta$-treated MMCs models.

\section{Methods}


Mouse mesangial cells (MMCs) were purchased from ATCC (CRL 1927), and the recombinant mouse transforming growth factor-beta (TGF- $\beta$ ) was obtained from R\&D Systems (USA). MMCs were grown at $37^{\circ} \mathrm{C}$ in plastic flasks in Dulbecco's Modified Eagle's medium/Ham's F12 Medium (DMEM/F12; 3:1 mixture; Invitrogen Corporation, Gaithersburg, MD, USA) containing 10\% fetal bovine serum (FBS), penicillin ( $50 \mathrm{U} / \mathrm{mL}$ ), and $2.6 \mathrm{~g}$ HEPES. The culture flasks were maintained in a $95 \% \mathrm{O}_{2}$ and $5 \% \mathrm{CO}_{2}$ humidified environment. At confluence, cells were exposed to DMEM/F12 medium containing no FBS for $24 \mathrm{~h}$ according to the following experimental groups: control, cultured in DMEM/F12 medium and TGF- $\beta$ group, cultured in DMEM/F12 containing $5 \mathrm{ng} / \mathrm{mL}$ of recombinant TGF- $\beta$. After $24 \mathrm{~h}$ of incubation, cells were rinsed twice with PBS, and $1 \mathrm{~mL}$ of the commercial kit (TRIzol, Gibco BRL, Rockland, MD, USA) was added to isolate total RNA and evaluate the mRNA expression.

\section{Housekeeping genes}

The selection of the candidate HKG was based on published studies that used RT-PCR for gene expressions in kidney diseases models. Then, five genes were selected (Actb, Hprt, Gapdh, $18 S$ and Ppia) being constitutively expressed in kidney cells with independent cellular functions.

\section{mRNA expression by RT-PCR}

The mRNA expression levels were estimated by quantitative RT-PCR. The total RNA was purified from MMCs using a TRIzol kit according to the manufacturer's instructions. A mass of $2 \mu \mathrm{g}$ of total RNA was treated with DNase (Promega, Madison, WI, USA) to prevent genomic DNA contamination. The RNA pellet was resuspended in RNase-free water and reverse transcribed into cDNA using a High-Capacity cDNA Reverse Transcription Kit (Applied Biosystems). RT-PCR amplification was performed using the QuantStudio (TM) 7 Flex System (Applied Biosystems), with specific primers for each gene as follows (sense and antisense, respectively): Fibronectin ( $5^{\prime \prime}$ acactaacgtaaattgccca $3^{\prime}$ and 5' gctaacatcactggggtgtggat $\left.3^{\prime}\right)$, Vimentin ( $5^{\prime}$ aggtggatcagctcaccaatgaca $3^{\prime}$ and $5^{\prime}$ tcaaggtcaagacgtgccagagaa $\left.3^{\prime}\right), a-S M A$ ( $5^{\prime}$ 'tattgtgctggactctggagatgg $3^{\prime}$ and $5^{\prime}$ agtagtcacgaaggaatagccacg $\left.3^{\prime}\right)$, Actb (5' cctcatgccaacacagtgc $3^{\prime}$ and $5^{\prime}$ acatctgctggaaggtggac $\left.3^{\prime}\right)$, Hprt (5' ctcatggactgattatggacaggac $3^{\prime}$ and 5' gcaggtcagcaaagaacttatagcc $\left.3^{\prime}\right)$, Gapdh (5' ggtggtctcctctgactttaaca $3^{\prime}$ and $5^{\prime}$ accaggaaatgagccttgacaaag $\left.3^{\prime}\right), 18 S$ (5' gactgtctcgccggtgtc $3^{\prime}$ and $5^{\prime}$ ggagagccggaacgtcga $3^{\prime}$ ) and Ppia (5' caggtccatctacggagaga $3^{\prime}$ and $5^{\prime}$ catccagccattcagtcttg $\left.3^{\prime}\right)$. The relative gene expression was calculated using the PCR conditions under which the amplification curve was logarithmic.

\section{Analysis of housekeeping gene expression stability}

To define the best housekeeping gene and the best combination, we evaluate the cycle thresholds (Ct) value of RT-PCR in five different software applications: DataAssist (version 3.1; https://www.thermofsher.com/br/en/home/technicalresources/sofware-downloads/dataassist-sofware.html), Bestkeeper (version 1.0; https://www.genequantifcation.de/bestkeeper.html), RefFinder and the comparative $\Delta$ Ct method (https://www.heartcure.com.au/refnder/), GeNorm (https://genorm.cmgg.be/), RefFinder and the comparative $\Delta$ Ct method (https://www.heartcure.com.au/refnder/), and NormFinder (version 0.953; https://moma.dk/normfndersofware), following the authors' recommendations. These software packages determine the relative expression stability of the candidate HKG and generate a rank of the best genes ${ }^{16}$. NormFinder is a freely available tool and recommends a standard deviation less than $0.5(\mathrm{SD}<0.5)$. GeNorm software calculates the gene stability measure (M value) and recommends that this value falls below $1.5(\mathrm{M}<1.5)$. BestKeeper evaluates the SD and coefficient of variation (CV) of the samples, and a SD of less than $1.0(\mathrm{SD}<1.0)$ and a CV of less than $3.0(\mathrm{CV}<3.0)$ are required. DataAssist shows the $\mathrm{Ct}$ values of the candidate genes for all samples and organizes them by score. The comparative $\triangle \mathrm{Ct}$ method was used to calculate the mean SD of the samples. RefFinder software includes all the above software and calculates the geometric mean (Geomean). The optimal number of HKG was evaluated using the GenEx software 
package, which calculates the accumulated standard deviation (Acc.SD) of sample groups and estimates the ideal number of genes for normalization. The following groups were included in the analysis, control, TGF- $\beta$-treated and All (control + TGF- $\beta$ samples).

\section{Statistical analysis}

The test of normality (Shapiro-Wilk test) showed that Ct values of HKG were not normally distributed; hence, the median values are expressed as horizontal lines, and the error bars represent interquartile range. Initially, all groups were analyzed using the Mann-Whitney $U$ test, after which, samples were analyzed using Spearman's correlation. A value between $0.30-0.50$ was determined as a weak correlation, $0.50-0.70$ as moderate, $0.70-0.90$ as strong and $0.90-$ 1.00 as very strong.

\section{Declarations}

\section{Acknowledgements}

This study was supported by grants from Fundação de Amparo à Pesquisa do Estado de São Paulo (FAPESP; MAB: 16/02185-6), Coordenação de Aperfeiçoamento de Pessoal de Nível Superior (CAPES), Conselho Nacional de Desenvolvimento Científico e Tecnológico (CNPq; Process: 439042/2018-6).

\section{Authors' contributions}

$B B, A C A$ and $A S N$ designed the study. BB, EM and ASN performed the experiments. BB and ACA analyzed the data. BB, MAB and ASN wrote the first draft of the manuscript. MAB and ASN provided financial support, study materials, and the final approval of the manuscript. All authors reviewed the final version of the manuscript.

\section{Data availability statement}

All data generated or analyzed during this study are included in this published article [and its supplementary information files].

\section{Competing interests}

The authors declare no competing interests.

\section{References}

1. Nogueira, A., Pires, M. J. \& Oliveira, P. A. Pathophysiological Mechanisms of Renal Fibrosis: A Review of Animal Models and Therapeutic Strategies. In Vivo 31, 1-22, doi:10.21873/invivo.11019 (2017).

2. Simonson, M. S. Phenotypic transitions and fibrosis in diabetic nephropathy. Kidney Int 71, 846-854, doi:10.1038/sj.ki.5002180 (2007).

3. Leask, A. \& Abraham, D. J. TGF-beta signaling and the fibrotic response. FASEB J 18, 816-827, doi:10.1096/fj.031273rev (2004).

4. Padgett, R. W. \& Reiss, M. TGFbeta superfamily signaling: notes from the desert. Development 134, 3565-3569, doi:10.1242/dev.005926 (2007).

5. Rahimi, R. A. \& Leof, E. B. TGF-beta signaling: a tale of two responses. J Cell Biochem 102, 593-608, doi:10.1002/jcb.21501 (2007). 
6. Derveaux, S., Vandesompele, J. \& Hellemans, J. How to do successful gene expression analysis using real-time PCR. Methods 50, 227-230, doi:10.1016/j.ymeth.2009.11.001 (2010).

7. Ho-Pun-Cheung, A. et al. Reverse transcription-quantitative polymerase chain reaction: description of a RIN-based algorithm for accurate data normalization. BMC Mol Biol 10, 31, doi:10.1186/1471-2199-10-31 (2009).

8. Bustin, S. A. et al. The MIQE guidelines: minimum information for publication of quantitative real-time PCR experiments. Clin Chem 55, 611-622, doi:10.1373/clinchem.2008.112797 (2009).

9. Schwarzenbach, H., da Silva, A. M., Calin, G. \& Pantel, K. Data Normalization Strategies for MicroRNA Quantification. Clin Chem 61, 1333-1342, doi:10.1373/clinchem.2015.239459 (2015).

10. Muñoz, J. J. et al. Identification of housekeeping genes for microRNA expression analysis in kidney tissues of Pkd1 deficient mouse models. Sci Rep 10, 231, doi:10.1038/s41598-019-57112-4 (2020).

11. Caracausi, M. et al. Systematic identification of human housekeeping genes possibly useful as references in gene expression studies. Mol Med Rep 16, 2397-2410, doi:10.3892/mmr.2017.6944 (2017).

12. Eisenberg, E. \& Levanon, E. Y. Human housekeeping genes, revisited. Trends Genet 29, 569-574, doi:10.1016/j.tig.2013.05.010 (2013).

13. Wang, Z., Lyu, Z., Pan, L., Zeng, G. \& Randhawa, P. Defining housekeeping genes suitable for RNA-seq analysis of the human allograft kidney biopsy tissue. BMC Med Genomics 12, 86, doi:10.1186/s12920-019-0538-z (2019).

14. Jung, M. et al. In search of suitable reference genes for gene expression studies of human renal cell carcinoma by real-time PCR. BMC Mol Biol 8, 47, doi:10.1186/1471-2199-8-47 (2007).

15. Huggett, J., Dheda, K., Bustin, S. \& Zumla, A. Real-time RT-PCR normalisation; strategies and considerations. Genes Immun 6, 279-284, doi:10.1038/sj.gene.6364190 (2005).

16. Hosni, N. D., Anauate, A. C. \& Boim, M. A. Reference genes for mesangial cell and podocyte qPCR gene expression studies under high-glucose and renin-angiotensin-system blocker conditions. PLoS One 16, e0246227, doi:10.1371/journal.pone.0246227 (2021).

17. Muñoz, J. J. et al. Ppia is the most stable housekeeping gene for qRT-PCR normalization in kidneys of three Pkd1deficient mouse models. Sci Rep 11, 19798, doi:10.1038/s41598-021-99366-x (2021).

18. Guan, Q., Nguan, C. Y. \& Du, C. Expression of transforming growth factor-beta1 limits renal ischemia-reperfusion injury. Transplantation 89, 1320-1327, doi:10.1097/TP.0b013e3181d8e9dc (2010).

19. Morrissey, J. et al. Transforming growth factor-beta induces renal epithelial jagged-1 expression in fibrotic disease. J Am Soc Nephrol 13, 1499-1508, doi:10.1097/01.asn.0000017905.77985.4a (2002).

20. Wu, X. et al. Exosomes from high glucose-treated glomerular endothelial cells trigger the epithelial-mesenchymal transition and dysfunction of podocytes. Sci Rep 7, 9371, doi:10.1038/s41598-017-09907-6 (2017).

21. Cappelli, C. et al. The TGF- $\beta$ profibrotic cascade targets ecto-5'-nucleotidase gene in proximal tubule epithelial cells and is a traceable marker of progressive diabetic kidney disease. Biochim Biophys Acta Mol Basis Dis 1866, 165796, doi:10.1016/j.bbadis.2020.165796 (2020).

22. Castro, N. E., Kato, M., Park, J. T. \& Natarajan, R. Transforming growth factor $\beta 1$ (TGF- $\beta 1$ ) enhances expression of profibrotic genes through a novel signaling cascade and microRNAs in renal mesangial cells. J Biol Chem 289, 29001-29013, doi:10.1074/jbc.M114.600783 (2014).

23. Takakura, K., Tahara, A., Sanagi, M., Itoh, H. \& Tomura, Y. Antifibrotic effects of pirfenidone in rat proximal tubular epithelial cells. Ren Fail 34, 1309-1316, doi:10.3109/0886022X.2012.718955 (2012).

24. Deshpande, S. et al. Reduced Autophagy by a microRNA-mediated Signaling Cascade in Diabetes-induced Renal Glomerular Hypertrophy. Sci Rep 8, 6954, doi:10.1038/s41598-018-25295-x (2018). 
25. Huang, F. et al. High glucose and TGF- $\beta 1$ reduce expression of endoplasmic reticulum-resident selenoprotein $S$ and selenoprotein $\mathrm{N}$ in human mesangial cells. Ren Fail 41, 762-769, doi:10.1080/0886022X.2019.1641413 (2019).

26. Jia, Y. et al. Dysregulation of histone $\mathrm{H} 3$ lysine 27 trimethylation in transforming growth factor- $\beta 1$-induced gene expression in mesangial cells and diabetic kidney. J Biol Chem 294, 12695-12707, doi:10.1074/jbc.RA119.007575 (2019).

27. Masola, V. et al. In vitro effects of interleukin (IL)-1 beta inhibition on the epithelial-to-mesenchymal transition (EMT) of renal tubular and hepatic stellate cells. J Transl Med 17, 12, doi:10.1186/s12967-019-1770-1 (2019).

28. Ma, J. et al. Up-regulation of microRNA-93 inhibits TGF- $\beta 1$-induced EMT and renal fibrogenesis by down-regulation of Orai1. J Pharmacol Sci 136, 218-227, doi:10.1016/j.jphs.2017.12.010 (2018).

29. Suzuki, Y. et al. Transforming growth factor- $\beta$ induces vascular endothelial growth factor-C expression leading to lymphangiogenesis in rat unilateral ureteral obstruction. Kidney Int 81, 865-879, doi:10.1038/ki.2011.464 (2012).

30. Biederman, J., Yee, J. \& Cortes, P. Validation of internal control genes for gene expression analysis in diabetic glomerulosclerosis. Kidney Int 66, 2308-2314, doi:10.1111/j.1523-1755.2004.66016.x (2004).

31. Leong, K. G., Ozols, E., Kanellis, J., Nikolic-Paterson, D. J. \& Ma, F. Y. Cyclophilin A Promotes Inflammation in Acute Kidney Injury but Not in Renal Fibrosis. Int J Mol Sci 21, doi:10.3390/ijms21103667 (2020).

32. Nigro, P., Pompilio, G. \& Capogrossi, M. C. Cyclophilin A: a key player for human disease. Cell Death Dis 4, e888, doi:10.1038/cddis.2013.410 (2013).

33. Ramachandran, S. et al. Plasma level of cyclophilin A is increased in patients with type 2 diabetes mellitus and suggests presence of vascular disease. Cardiovasc Diabetol 13, 38, doi:10.1186/1475-2840-13-38 (2014).

34. Nicholls, C., Li, H. \& Liu, J. P. GAPDH: a common enzyme with uncommon functions. Clin Exp Pharmacol Physiol 39, 674-679, doi:10.1111/j.1440-1681.2011.05599.x (2012).

35. Seidler, N. W. GAPDH and intermediary metabolism. Adv Exp Med Biol 985, 37-59, doi:10.1007/978-94-007-4716$6 \_2(2013)$.

36. Tarze, A. et al. GAPDH, a novel regulator of the pro-apoptotic mitochondrial membrane permeabilization. Oncogene 26, 2606-2620, doi:10.1038/sj.onc.1210074 (2007).

37. Meyer, A., Todt, C., Mikkelsen, N. T. \& Lieb, B. Fast evolving 18S rRNA sequences from Solenogastres (Mollusca) resist standard PCR amplification and give new insights into mollusk substitution rate heterogeneity. BMC Evol Biol 10, 70, doi:10.1186/1471-2148-10-70 (2010).

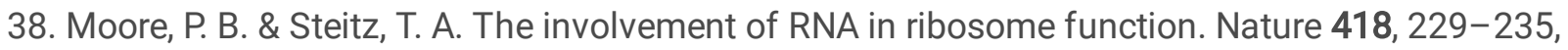
doi:10.1038/418229a (2002).

39. Kang, T. H., Park, Y., Bader, J. S. \& Friedmann, T. The housekeeping gene hypoxanthine guanine phosphoribosyltransferase (HPRT) regulates multiple developmental and metabolic pathways of murine embryonic stem cell neuronal differentiation. PLoS One 8, e74967, doi:10.1371/journal.pone.0074967 (2013).

40. Townsend, M. H., Robison, R. A. \& O'Neill, K. L. A review of HPRT and its emerging role in cancer. Med Oncol 35, 89, doi:10.1007/s12032-018-1144-1 (2018).

41. Bugyi, B. \& Kellermayer, M. The discovery of actin: "to see what everyone else has seen, and to think what nobody has thought". J Muscle Res Cell Motil 41, 3-9, doi:10.1007/s10974-019-09515-z (2020).

42. Schnaper, H. W., Hayashida, T., Hubchak, S. C. \& Poncelet, A. C. TGF-beta signal transduction and mesangial cell fibrogenesis. Am J Physiol Renal Physiol 284, F243-252, doi:10.1152/ajprenal.00300.2002 (2003).

43. Bjerregaard, H., Pedersen, S., Kristensen, S. R. \& Marcussen, N. Reference genes for gene expression analysis by real-time reverse transcription polymerase chain reaction of renal cell carcinoma. Diagn Mol Pathol 20, 212-217, doi:10.1097/PDM.0b013e318212e0a9 (2011). 
44. Cui, X., Zhou, J., Qiu, J., Johnson, M. R. \& Mrug, M. Validation of endogenous internal real-time PCR controls in renal tissues. Am J Nephrol 30, 413-417, doi:10.1159/000235993 (2009).

45. Schmid, H. et al. Validation of endogenous controls for gene expression analysis in microdissected human renal biopsies. Kidney Int 64, 356-360, doi:10.1046/j.1523-1755.2003.00074.x (2003).

46. Ma, Y., Dai, H., Kong, X. \& Wang, L. Impact of thawing on reference gene expression stability in renal cell carcinoma samples. Diagn Mol Pathol 21, 157-163, doi:10.1097/PDM.0b013e31824d3435 (2012).

47. Ma, Y. et al. Renal tissue thawed for 30 minutes is still suitable for gene expression analysis. PLoS One 9, e93175, doi:10.1371/journal.pone.0093175 (2014).

48. Zinzow-Kramer, W. M., Horton, B. M. \& Maney, D. L. Evaluation of reference genes for quantitative real-time PCR in the brain, pituitary, and gonads of songbirds. Horm Behav 66, 267-275, doi:10.1016/j.yhbeh.2014.04.011 (2014).

49. Barber, R. D., Harmer, D. W., Coleman, R. A. \& Clark, B. J. GAPDH as a housekeeping gene: analysis of GAPDH mRNA expression in a panel of 72 human tissues. Physiol Genomics 21, 389-395, doi:10.1152/physiolgenomics.00025.2005 (2005).

50. Gholami, K., Loh, S. Y., Salleh, N., Lam, S. K. \& Hoe, S. Z. Selection of suitable endogenous reference genes for qPCR in kidney and hypothalamus of rats under testosterone influence. PLoS One 12, e0176368, doi:10.1371/journal.pone.0176368 (2017).

51. Bas, A., Forsberg, G., Hammarström, S. \& Hammarström, M. L. Utility of the housekeeping genes 18S rRNA, betaactin and glyceraldehyde-3-phosphate-dehydrogenase for normalization in real-time quantitative reverse transcriptase-polymerase chain reaction analysis of gene expression in human T lymphocytes. Scand J Immunol 59, 566-573, doi:10.1111/j.0300-9475.2004.01440.x (2004).

52. Banda, M., Bommineni, A., Thomas, R. A., Luckinbill, L. S. \& Tucker, J. D. Evaluation and validation of housekeeping genes in response to ionizing radiation and chemical exposure for normalizing RNA expression in real-time PCR. Mutat Res 649, 126-134, doi:10.1016/j.mrgentox.2007.08.005 (2008).

53. Granfar, R. M., Day, C. J., Kim, M. S. \& Morrison, N. A. Optimised real-time quantitative PCR assays for RANKL regulated genes. Mol Cell Probes 19, 119-126, doi:10.1016/j.mcp.2004.10.003 (2005).

\section{Figures}



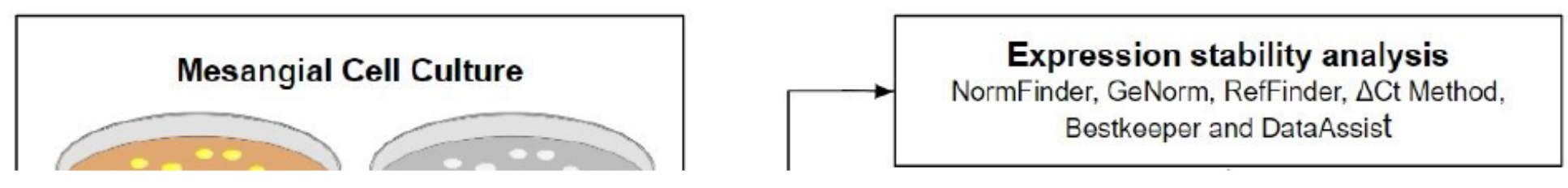

Figure 1

Workflow diagram illustrating the strategy for identification of housekeeping normalizer for RT-PCR. Actb, Hprt, Gapdh, $18 S$ and Ppia housekeeping genes (HKG) selected from the literature for comparison.

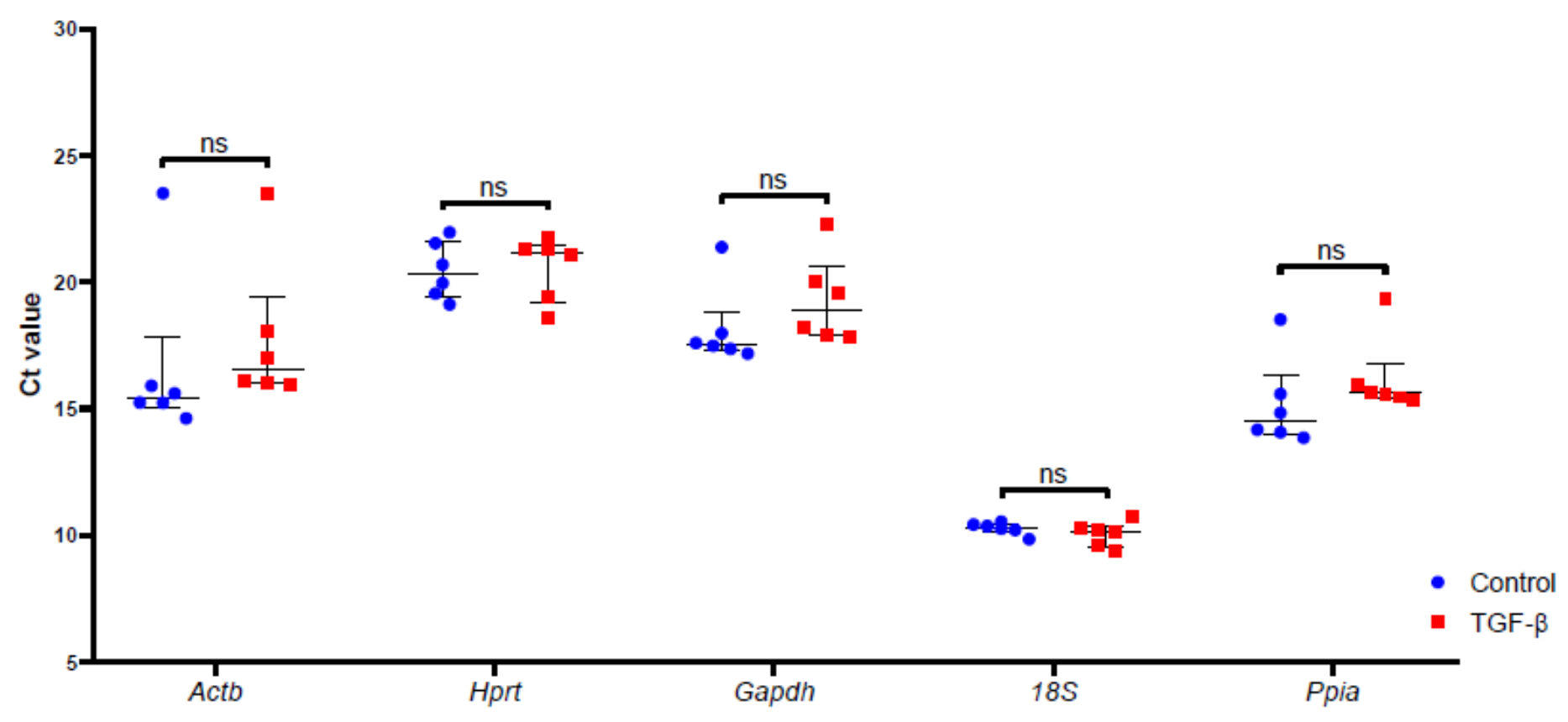

Figure 2 
Ct values of five candidate housekeeping genes. A lower cycle threshold (Ct) value indicates a higher gene expression. The median values are expressed as horizontal lines, and the error bars represent the interquartile range. The $\mathrm{Ct}$ values of $18 S$ were the lowest, indicating the highest expression levels. ns, non-significant.

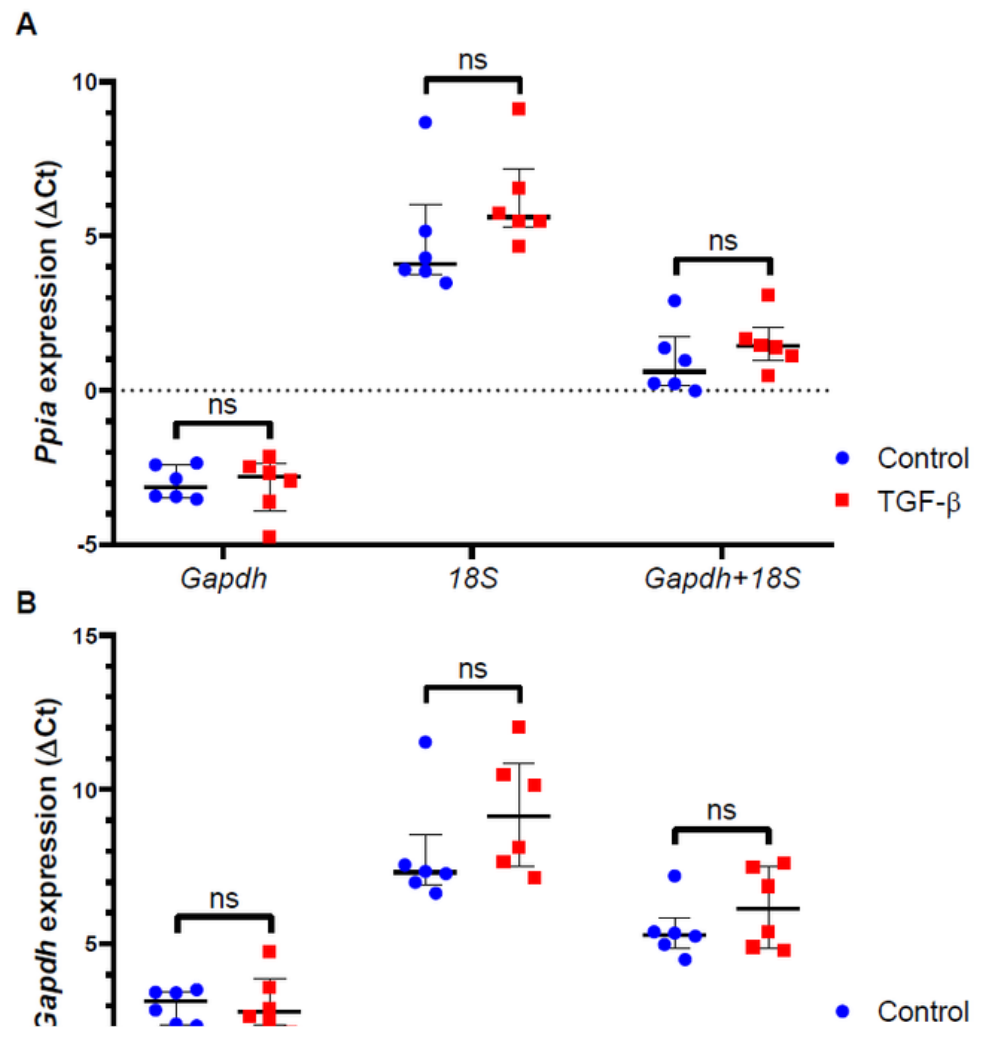

\section{Figure 3}

The $\Delta$ Ct values of Ppia (A), Gapdh (B) and $18 S$ (C) candidate housekeeping genes were normalized by combinations of each other. A lower Ct value indicates a higher gene expression. The median values are expressed as horizontal lines, and the error bars represent the interquartile range. Ppia, target expression normalized by Gapdh, $18 S$ and Gapdh+18S, Gapdh, target expression normalized by Ppia, $18 S$ and Ppia+18S, 18S, target expression normalized by Ppia, Gapdh and Ppia+Gapdh. ns, non-significant. 


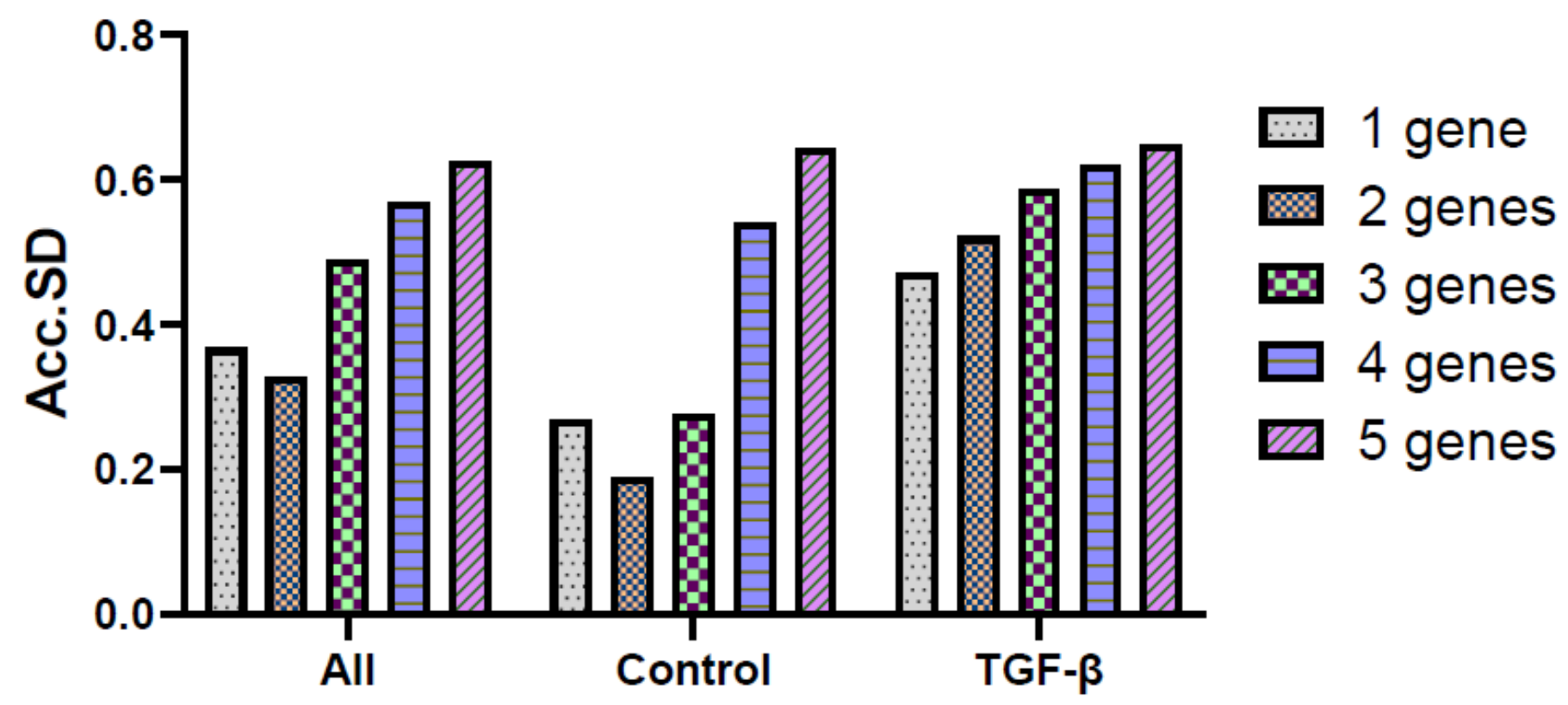

Figure 4

Optimal number of housekeeping genes according to GenEx software analysis. Accumulated standard deviation (Acc.SD) for the five candidate reference genes in all groups to estimate the ideal number of genes for normalization. Lower values of Acc.SD indicate the optimal number of reference genes. All, all samples.

Figure 5

Correlation matrix between the expression of Ppia and Gapdh (A), Ppia and 18S(B) and Gapdh and 18S(C) candidate housekeeping genes. $\rho$ : Spearman's rank correlation coefficient. ${ }^{*} \mathrm{p}<0.05$. 

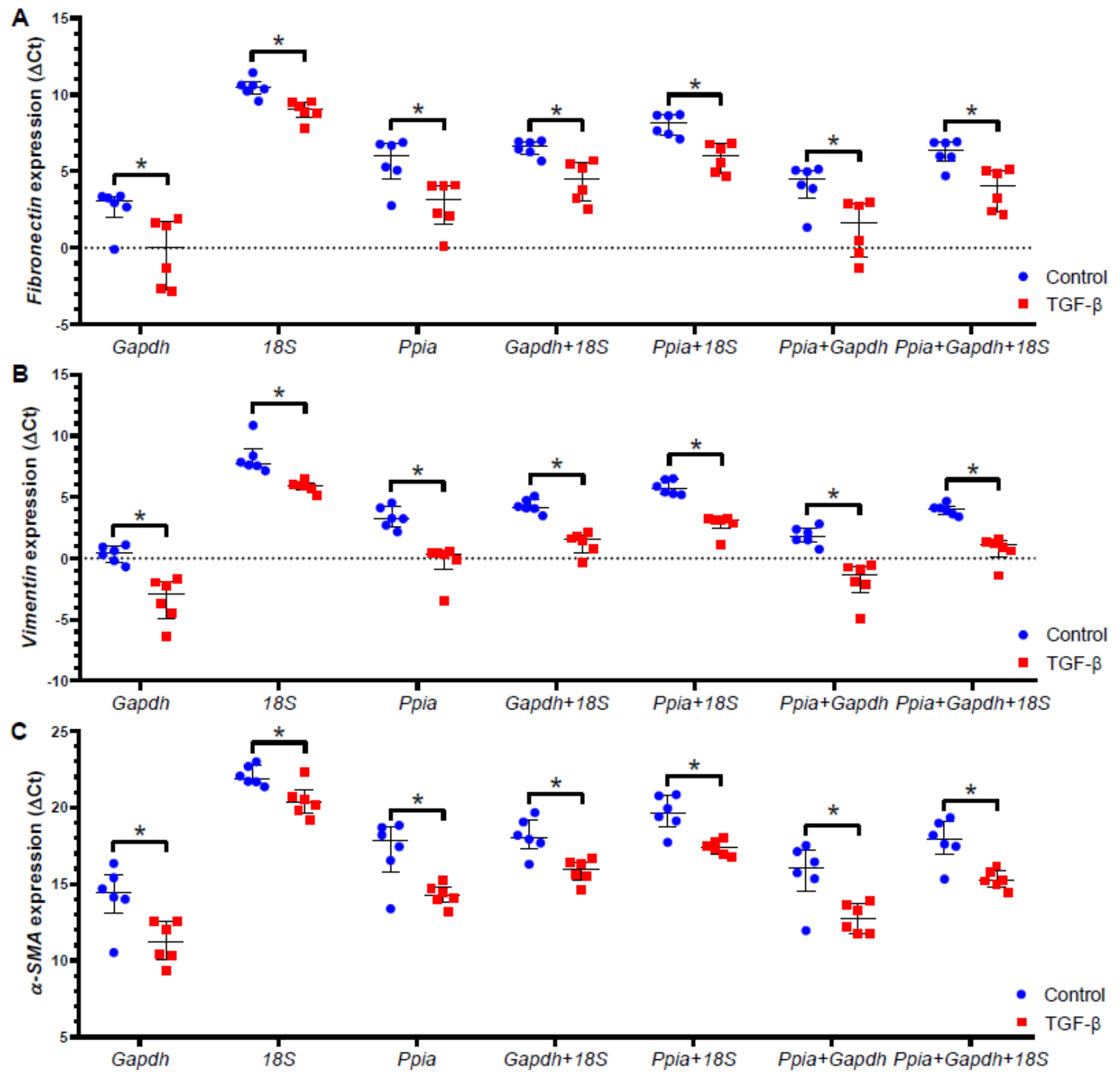

Figure 6

The $\triangle$ Ct values of fibronectin $(\mathbf{A})$, vimentin $(\mathbf{B})$, and $a-S M A(\mathbf{C})$ target genes normalized different combinations of the three best candidate housekeeping genes (Ppia, Gapdh, and 18S). A lower Ct value indicates a higher gene expression. The median values are expressed as horizontal lines, and the error bars represent the interquartile range. $p<0.05$ by Mann-Whitney U test: * vs control group.

\section{Supplementary Files}

This is a list of supplementary files associated with this preprint. Click to download. 
- AdditionalFile1.xlsx

- SupplementaryFigure1.pdf

Page 15/15 